\title{
On the Generalized Saletan Contractions
}

\author{
MARIAN KuPCZYŃSKI \\ Institute of Theoretical Physics, Warsaw University \\ Received February 18, 1969
}

\begin{abstract}
The generalized Saletan contractions leaving some subalgebra unchanged are investigated. By use of a simple basis the necessary and sufficient conditions for these contractions and the brackets of the contracted algebra expressed only by the structure constants of the algebra to be contracted are obtained. Many basis independent results are derived. Some of them give as a special case Saletan results. Similar contractions but for $\varepsilon \rightarrow \infty$ are proposed and investigated.
\end{abstract}

\section{Introduction}

The continuous process deforming one Lie group into another nonisomorphic Lie group is believed to have many physical applications. One postulates $[1,2]$ that some physical processes: like switching off interactions or passing to the limit with some physical quantities allowing to disregard some physical effects (for example velocity of light $\nearrow \infty$ ) should be reflected in the group theoretical description of the physical systems by some continuous deforming processes called contractions.

From many possible mathematical formulations of group-contraction [1-7] we choose the following one:

Let us consider $N$-dimensional Lie algebra $R^{(0)}$ on the vector space $S$. 'The corresponding Lie brackets will be denoted by $[,]^{(0)}$. We say that $R^{(0)}$ can be contracted by $A(\varepsilon)$ if there exists an analytic curve in a vector space $L(S, S)$ of all linear mappings of $S$ :

$$
E^{1} \ni \varepsilon \rightarrow A(\varepsilon) \in L(S, S)
$$

such that for $\varepsilon=\varepsilon_{1}-A\left(\varepsilon_{1}\right)=I$ and for all $\varepsilon \neq \varepsilon_{0}-A(\varepsilon)$ are one to one mappings of $S$ such that for all " $\boldsymbol{a}$ " $\in S$ and " $\boldsymbol{b}$ " $\in S$ there exists a limit, as $\varepsilon \rightarrow \varepsilon_{0}$, of series $[\boldsymbol{a}, \boldsymbol{b}]^{(0)}(\varepsilon)$ where

$$
\begin{aligned}
{[\boldsymbol{a}, \boldsymbol{b}]^{(0)}(\varepsilon) } & =A(\varepsilon)^{-1}[A(\varepsilon) \boldsymbol{a}, A(\varepsilon) \boldsymbol{b}]^{(0)}, \\
{[\boldsymbol{a}, \boldsymbol{b}]^{(1)} } & =\lim _{\varepsilon \rightarrow \varepsilon_{0}}[\boldsymbol{a}, \boldsymbol{b}]^{(0)}(\varepsilon) .
\end{aligned}
$$

The limit $[\boldsymbol{a}, \boldsymbol{b}]^{(1)}$ defines new Lie algebra $R^{(1)}$ on $S$ called a contraction of $R^{(0)}$. Usually one takes $\varepsilon_{0}=0$ or $\varepsilon_{0}=\infty$. If one chooses a basis in $S$ one obtains a representation of $A(\varepsilon)$ by a set of $(N \times N)$-matrices and 
the series (1) has a corresponding one for the structure constants of $R^{(0)}(\varepsilon)$ :

$$
C_{(0) \alpha, \beta}^{\gamma}(\varepsilon)=A^{-1}(\varepsilon)_{\gamma^{\prime}}^{\gamma} C_{(0) \alpha^{\prime}, \beta^{\prime}}^{\gamma^{\prime}} A(\varepsilon)_{\beta}^{\beta^{\prime}} A(\varepsilon)_{\alpha}^{\alpha^{\prime} \cdot{ }^{1}}
$$

The necessary and sufficient conditions for this contraction [4] one obtains demanding the existence of the limit of the series (3)

$$
C_{(1) \alpha, \beta}^{\gamma}=\lim _{\varepsilon \rightarrow \varepsilon_{0}} C_{(0) \alpha, \beta}^{\gamma}(\varepsilon) \text {. }
$$

The problems of deformations of Lie algebras are investigated in their full generality by means of modern differential geometry but the general investigations give the general results, which [5-7] are difficult sometimes to apply to the practical problems, so many simple realizations of the contraction have been proposed. Though they have been presented in different ways, all of them can be investigated by the use of Eq. (4).

Now we adopt the following notation: $\oplus$ is used for the direct sum of vector spaces and for the direct sum of linear operators or matrices for example: an operator $A=A_{1} \oplus A_{2}$ acts in a vector space $S=S_{1} \oplus S_{2}$ in a way $A S=A_{1} S_{1} \oplus A_{2} S_{2} ; \theta$ is used in the following meaning $\left(S \ominus S_{1}\right) \oplus S_{1}=S ; \widehat{\boldsymbol{a}, \boldsymbol{b}} \in S$ means for all " $\boldsymbol{a}$ " and " $\boldsymbol{b}$ "-elements of $S$.

We obtain the Inonü-Wigner contraction [3] if we choose

$$
\varepsilon_{0}=0, \quad A(\varepsilon)=(I+\varepsilon w) \oplus \varepsilon I
$$

where " $w$ " is a nonsingular matrix.

The Saletan contraction [4] is obtained by the choice:

$$
\varepsilon_{0}=0, \quad A(\varepsilon)=u+\varepsilon I, \quad u=u_{R} \oplus u_{N}
$$

where $u_{R}$ is a nonsingular matrix and $u_{N}$ is a nilpotent one of a rank $q$.

The generalized Saletan contractions one obtains [6] by taking

$$
A(\varepsilon)=\varepsilon^{p} A_{s}(\varepsilon)
$$

where $A_{s}(\varepsilon)$ is defined by $(6)$ and $p$ is a natural number.

The $p$-contractions $[1,8]$ are obtained by taking

$$
\varepsilon_{0}=0, \quad A(\varepsilon)_{j}^{i}=\varepsilon^{m_{i}} \delta_{j}^{i}, \quad 1 \leqq i, j \leqq N
$$

where for $m_{i}$ can be chosen real numbers ${ }^{2}$.

We are interested in the generalized Saletan contractions (7) which will be denoted $(S, p)$ and $(S, 0)$ if $p=0$.

For $(S, p)$ Eq. (1) and (2) were investigated for $A(\varepsilon)$ in the general form (6) and (7), Saletan obtained the necessary and sufficient conditions for $(S, 0)$ in the form of the general equations:

$$
\widehat{\boldsymbol{a}, \boldsymbol{b}} \in S \quad u^{2}[\boldsymbol{a}, \boldsymbol{b}]_{N}^{(0)}-u[u \boldsymbol{a}, \boldsymbol{b})_{N}^{(0)}-u[\boldsymbol{a}, u \boldsymbol{b}]_{N}^{(0)}+[u \boldsymbol{a}, u \boldsymbol{b}]_{N}^{(0)}=0
$$

1 We use Einstein summation convention $\sum_{i} a_{i} b_{i}=a^{i} b_{i}$.

${ }^{2}$ We use in this paper $\delta_{j}^{i}$ and $\delta_{i j}$ for Kronecker $\delta^{\prime} s$. 
and the new bracket in the form:

$\widehat{\boldsymbol{a}, \boldsymbol{b}} \in S \quad[\boldsymbol{a}, \boldsymbol{b}]^{(1)}=u^{-1}[u \boldsymbol{a}, u \boldsymbol{b}]_{R}^{(0)}-u[\boldsymbol{a}, \boldsymbol{b}]_{N}^{(0)}+[u \boldsymbol{a}, \boldsymbol{b}]_{N}^{(0)}+[\boldsymbol{a}, u \boldsymbol{b}]_{N}^{(0)}$,

where $[\boldsymbol{a}, \boldsymbol{b}]_{N}^{(0)}$ and $[\boldsymbol{a}, \boldsymbol{b}]_{R}^{(0)}$ are projections of $[\boldsymbol{a}, \boldsymbol{b}]^{(0)}$ into subspaces $S_{N}$ and $S_{R}, S_{N} \oplus S_{R}=S$. For $(S, p)$ Monique Levy-Nahas obtained more complicated results: new bracket is given by

$$
\widehat{\boldsymbol{a}, \boldsymbol{b}} \in S \quad[\boldsymbol{a}, \boldsymbol{b}]_{p}^{(1)}=(-u)^{p-1}\left([u \boldsymbol{a}, u \boldsymbol{b}]^{(0)}-u[\boldsymbol{a}, \boldsymbol{b}]^{(1)}\right)
$$

and the necessary and sufficient condition by

$$
u[\boldsymbol{a}, \boldsymbol{b}]_{p}^{(1)}=0
$$

where $p \geqq 1$ and $[\boldsymbol{a}, \boldsymbol{b}]^{(1)}$ is defined by $(10)$. For $(S, 0)$ Saletan found some simple corollaries expressible in the group theoretical language. For $(S, p)$ the same proofs seem to be very difficult.

\section{The Construction of the Basis}

We are going to investigate $(S, p)$ in a special basis which enables us to find the necessary and sufficient conditions for $(S, p)$ involving only the structure constants of $R^{(0)}$. To simplify calculations we put $u_{R}=I$ in (6), for doing it we have two arguments: $u_{R}$ being nonsingular transforms a subalgebra generated by $S_{R}$ in $R^{(0)}$ into an isomorphic subalgebra in $R^{(1)}$ so by our choice we don't loose much of generality. Besides for physical reasons we want to have a model fo contraction leaving some subalgebra unchanged.

Now we construct our basis in $S$, from matrix theory it is known that every nilpotent matrix $u_{N}$ of rank $q$ can be written in a very simple form in a basis consisting of all independent Jordan chains in $S_{Y}$, where Jordan chain generated by $\boldsymbol{a} \in S_{N}$ is a set of linearly independent vectors: $\boldsymbol{a}, u_{N} \boldsymbol{a}, u_{N}^{2} \boldsymbol{a}, \ldots u_{N}^{n_{a}-1} \boldsymbol{a}\left(n_{a} \leqq q, u_{N}^{n_{a}} \boldsymbol{a}=0\right)$. In this basis $S_{N}=\bigoplus_{a} S_{n_{a}}$, $\sum_{a} n_{a}=q_{1}[9]$, where $S_{n_{a}}$ is a vector space spanned by Jordan chain generated by " $\boldsymbol{a}$ ". The elements of our new basis will be denoted by $I_{l k}$. They are defined as follows:

$$
\begin{gathered}
u_{N}^{k} I_{l s}=I_{l s+k}, \quad I_{l l+1}=0 \\
1 \leqq s \leqq l, \quad l=l_{1}, \quad l_{2} \ldots l_{p}, \quad \sum_{i=1}^{p} l_{i}=q_{1} \cdot{ }^{3}
\end{gathered}
$$

To have all our basis in $S$ we add $N-q_{1}$ linearly independent vectors in $S_{R}$ which we signify $I_{s}, s=1, \ldots, N-q_{1}$. Now we let $I_{l k}$ and $I_{s}$ to

${ }^{3}$ Here $l_{i}$ is a dimension of vector space spanned by Jordan chain generated by vector $I_{l_{i} 1}$. 
satisfy the following commutation relations in $R^{(0)}$ :

$$
\begin{aligned}
{\left[I_{s}, I_{t}\right]^{(0)} } & =C_{(0) s, t}^{n} I_{n}+C_{(0) s, t}^{w i} I_{w i}, \\
{\left[I_{s}, I_{l k}\right]^{(0)} } & =C_{(0) s, l k}^{n} I_{n}+C_{(0) s, l k}^{w i} I_{w i}, \\
{\left[I_{r s}, I_{l k}\right]^{(0)} } & =C_{(0) r s, l k}^{n} I_{n}+C_{(0) r s, l k}^{w i} I_{w i} .
\end{aligned}
$$

\section{The Main Results}

The contracting series of the matrices $A(\varepsilon)=\varepsilon^{p}\left(\left(I \oplus u_{\nu}\right)+\varepsilon I\right)$ acts on our basis in the following way: $A(\varepsilon) I_{l k}=\varepsilon^{p} I_{l k+1}$ for $k \leqq l-1$, $A(\varepsilon) I_{l l}=\varepsilon^{p+1} I_{l l}, A(\varepsilon) I_{s}=\varepsilon^{p} I_{s}$ so $A(\varepsilon)_{\beta}^{\alpha}$ matrix, acting on the vectors in $S$ in our basis, has the form:

$A(\varepsilon)_{s}^{s^{\prime}}=\delta_{s}^{s^{\prime}} \varepsilon^{p}, \quad A(\varepsilon)_{w i}^{w^{\prime} i^{\prime}}=\delta_{w}^{w^{\prime}}\left(\delta_{i+1}^{i^{\prime}}+\varepsilon \delta_{i}^{i^{\prime}}\right) \varepsilon^{p}$,

$A^{-1}(\varepsilon)_{s^{\prime}}^{s}=\delta_{s^{\prime}}^{s} \varepsilon^{-p}, \quad A^{-1}(\varepsilon)_{w^{\prime} i^{\prime}}^{w i}=\delta_{w^{\prime}}^{w}(-1)^{i-i^{\prime}} \varepsilon^{-\left(i-i^{\prime}+1+p\right)}$ where $i \geqq i^{\prime}$.

Using (15) from (3) we obtain

$$
\begin{aligned}
& C_{(0) s, t}^{n}(\varepsilon)=\varepsilon^{p} C_{(0) s, t}^{n}, C_{(0) s, t}^{w i}(\varepsilon)=C_{(0) s, t}^{w i^{\prime}}(\varepsilon) \frac{(-1)^{i-i^{\prime}}}{\varepsilon^{i-i^{\prime}+1-p}},{ }^{4} \\
& C_{(0) s, l k}^{n}(\varepsilon)=\left(C_{(0) s, l k+1}^{n}+\varepsilon C_{(0) s, l k}^{n}\right) \varepsilon^{p}, \\
& C_{(0) s, l k}^{w i}(\varepsilon)=\left(C_{(0) s, l k+1}^{w i^{\prime}}+\varepsilon C_{(0) s, l k}^{w i^{\prime}}\right) \frac{(-1)^{i-\imath^{\prime}}}{\varepsilon^{i-i^{\prime}+1-p}}, \\
& C_{(0) r s, l k}^{n}(\varepsilon)=\left(C_{(0) r s+1, l k+1}^{n}+\varepsilon C_{(0) r s+1, l k}^{n}\right. \\
& \left.+\varepsilon C_{(0) r s, l k+1}+\varepsilon^{2} C_{(0) r s, l k}^{n}\right) \varepsilon^{p}, \\
& C_{(0) r s, l k}^{w i}(\varepsilon)=\left(C_{(0) r s+1, l k+1}^{w i^{\prime}}+\varepsilon C_{(0) r s+1, l k}^{w i^{\prime}}\right. \\
& \left.+\varepsilon C_{(0) r s, l k+1}^{w i^{\prime}}+\varepsilon^{2} C_{(0) r s, l k}^{w i^{\prime}}\right) \frac{(-1)^{i-i^{\prime}}}{\varepsilon^{i-i^{\prime}+1-p}} .
\end{aligned}
$$

From the Eq. (16), demanding all coefficients standing at $\varepsilon^{-k}$ for $k=1,2$, ... to vanish, we obtain:

Theorem 1. The algebra $R^{(0)}$ can be contracted by $(S, p)$ if and only if there exists a basis in $R^{(0)}$ such that the structure constants of $R^{(0)}$ in this basis fulfil the following equations:

$$
\begin{aligned}
C_{(0) s, l k+1}^{w i}-C_{(0) s, l k}^{w i-1} & =0, \\
C_{(0) s, t}^{w i} & =0, \\
C_{(0) r s+1, l k+1}^{w i}-C_{(0) r s+1, l k}^{w i-1}-C_{(0) r s, l k+1}^{w i-1}+C_{(0) r s, l k}^{w i-2} & =0
\end{aligned}
$$

for $1 \leqq i \leqq w-p=N_{1}, 1 \leqq s, t \leqq N-q_{1}, r 1 \leqq r s \leqq r r, l 1 \leqq l k \leqq l l$, we assume that $C_{(0) \beta, l l+1}^{\gamma}=C_{(0) \beta, l 0}^{\gamma}=C_{(0) \beta, \alpha}^{w_{0} 0+1}=C_{(0) \beta, \alpha}^{w 0}=0$ where $\alpha$, $\beta, \gamma$ are arbitrary indices labelling elements of the basis.

Now assuming that $(17-19)$ are fulfilled we read from (16) the structure constants of $R^{(1)}$.

${ }^{4}$ Here and in the following formulae we have summation over $i^{\prime}$ from 1 to $i$. 
Theorem 2. There exists a basis in the contracted algebra $R^{(1)}$ that the structure constants of $R^{(1)}$ in this basis are given by the following equalities:

$$
\begin{aligned}
& C_{(1) s, t}^{n}=\delta_{p 0} C_{(0) s, t}^{n}, \quad C_{(1) s, t}^{w i}=\delta_{i w} \delta_{p 1}(-1)^{p-1} C_{(0) s, t}^{w i+1-p}, \\
& C_{(1) s, l k}^{n}=\delta_{p 0} C_{(0) s, l k+1}^{n}, C_{(1) s, l k}^{w i}=\delta_{p 0} C_{(0) s, l k}^{w i} \quad \text { for } \quad i \leqq w-1 \text {, } \\
& C_{(1) s, l k}^{w w}=(-1)^{p-1}\left[\left(1-\delta_{p 0}\right) C_{(0) s, l k+1}^{w w+1-p}-C_{(0) s, l}^{w w}\right] \text {, } \\
& C_{(1) r s, l k}^{n}=\delta_{p 0} C_{(0) r s+1, l k+1}^{n}, C_{(0) r s, l k}^{w i}=\delta_{p 0} C_{(0) r s+1, l k+1}^{w i+1} \\
& \text { for } i \leqq w-1 \text {, } \\
& C_{(1) r s, l k}^{w w}=(-1)^{p-1}\left[C_{(0) r s+1}^{w w+1-p}, l k+1\left(1-\delta_{p 0}\right)-C_{(0) r s+1, l k}^{w w-p}\right. \\
& \left.-C_{(0) r s, l k+1}^{w w-p}+C_{(0) r s, l k-1}^{w w-p-1}\right]
\end{aligned}
$$

in all these equations we have no summation over $p$.

Now we are going to derive a few corollaries. Some of them will be basis independent, for this reason we need the following definitions. We define null spaces for $u^{i *}$ by $S_{i}\left(S_{i}\right.$ are spanned by vectors $I_{l k}$ for $l-i+1 \leqq k \leqq l$. By $A_{i}$ we will signify vector spaces spanned by $I_{l l-i+1}: A_{i}=S_{i} \ominus S_{i-1}$, finally we remark that vector spaces $u^{i} S$ are spanned by all vectors $I_{s}$ and $I_{l k}$ for $k>i$.

Corollary 1. All vector spaces $u^{i} S+S_{p}, * *$ for $i=1 \ldots q$, have to form subalgebras in $R^{(0)}$.

Corollary 2. All vector spaces $A_{i}$ and $A_{j}$ have to satisfy the following commutation relations: $\left[A_{i}, A_{j}\right]^{(0)} \subset S_{i+j+p}$.

Corollary 3. For $p \neq 0$ one obtains condition $[S, S]^{(1)} \subset S_{1}$ what is Monique Nahas condition for $(S, p)$.

Corollary 4. $R^{(1)}$ can be contracted by the same $A(\varepsilon)$ once more, if $p>1$ one obtains an abelian algebra $R^{(2)}$; if $p=0$ one obtains a series of contracted algebras $R^{(i)}$ where $R^{(i)}$ is obtained from $R^{(i-1)}$ by $A(\varepsilon) 1 \leqq i \leqq q$, the structure constants of $R^{(m)}$ are given by the following equalities:

$$
\begin{aligned}
C_{(m) s, t}^{n} & =C_{(0) s, t}^{n}, C_{(m) s, t}^{w i}=0, \\
C_{(m) s, l k}^{n} & =C_{(0) s, l k+m}^{n}, C_{(m) s, l k}^{w i}=C_{(0) s, l k}^{w i}, \\
C_{(m) r s, l k}^{n} & =C_{(0) r s+m, l k+m}^{n}, C_{(m) r s, l k}^{w i}=C_{(0) r s+m, l k+m}^{w i+m} \quad \text { for } \quad i \leqq w-m, \\
C_{(m) r s, l k}^{w w-c} & =C_{(0) r s+m, l k+c}^{w w}+C_{(0) r s+c, l k+m-C_{(0) r s+c, l k+c}^{w w} w+c}^{w w}+\text { for } \quad 0 \leqq c<m ;
\end{aligned}
$$

if $p=1$ one also obtains a series of contracted algebras $R_{1}^{(i)}$, for $i \leqq q$, which is different from the above mentioned one, the non vanishing structure constants of $R_{1}^{(m)}$ are following

$$
\begin{aligned}
C_{1(m) s, t}^{w w} & =C_{(0) \varepsilon, t}^{w w}, \\
C_{1(m) s, l k}^{w w} & =C_{(0) s, l k+m}^{w w}, \\
C_{1(m) r s, l k}^{w w} & =C_{(0) r s+m, l k+m}^{w w}
\end{aligned}
$$

* $u^{i}-$ means $u$ taken to the $i$-th power.

** + - here means vector sum of subspaces of $S$. 
where from the following basis independent features of $R_{1}^{(m)}$ can be found:

$$
\begin{aligned}
{\left[S_{i}, S\right]_{1}^{(m)} } & =0 \text { for } m \geqq i, \\
{\left[S_{R}, S_{R}\right]_{1}^{(m)} } & =\left[S_{R}, S_{R}\right]_{1}^{(1)} \subset S_{1} \text { for all } m,
\end{aligned}
$$

where []$_{1}^{(m)}$ denotes the bracket of $R_{1}^{(m)}$.

Corollary 5. All vector spaces $u^{i} S$ form subalgebras in all $R^{(m)}$.

Corollary 6. All vector spaces $A_{i}$ satisfy the following commutation relations $\left[A_{i}, A_{j}\right]^{(m)} \subset S_{i-m+j}$ for $m \geqq i$ and $m \geqq j$.

Corollary 7. All spaces $S_{k}$ satisfy the following relations:

$$
\left[S_{k}, S_{k}\right]^{(k+l)} \subset S_{k-\imath} \text { for } l=0,1 \ldots
$$

Corollary 8. The vector spaces $S_{k}$ form ideals in all $R^{(m)}$ for $m \geqq k$ and abelian ideals for $m \geqq 2 k$.

\section{The Derivation of the Corollaries}

At first we derive some simple corollaries of $(17-19)$ expressed by the structure constants of $R^{(0)}$ :

a) $C_{(0) s, l k}^{w i}=C_{(0) s, l 1}^{w i-k+1}$ for $i \geqq k, 5$

b) $C_{(0) s, l k}^{w o}=0$ for $i<k$,

c) $C_{(0) s, l l}^{w o i-1}=0$,

d) $C_{(0) s, l l-k}^{w i-k-1}=0$,

e) $C_{(0) r k+1, l k+1}^{w 1}=0$

f) $C_{(0) r s+1, l k+1}^{w i}=C_{(0) r s+1, l 1}^{w i-k}$ for $s \geqq i>k$,

g) $C_{(0) r}^{w i} r+1, l k+1=0$ for $s, k \geqq i$,

h) $C_{(0) r s+1, l k+1}^{w i}=C_{(0) r 1, l k+1}^{w i-s}$ for $k \geqq i>s$,

i) $C_{(0) r s+1, l k+1}^{w i}-C_{(0) r s+1, l k}^{w i-1}=C_{(0) r 1, l k+1}^{w i-s}-C_{(0) r 1, l k}^{w i-s-1}$ for $i>s$,

j) $C_{(0) r s+1, l k+1}^{w}-C_{(0) r s, l k+1}^{w i-1}=C_{(0) r s+1, l 1}^{w i-k}-C_{(0) r s, l 1}^{w i-k}-1$ for $i>k$,

k) $C_{(0) r r-k, l l}^{w i-1}=C_{(0) r r-k, l l-1}^{w o i-2}, C_{(0) r r-k, l l}^{w, i-2}=0$

for $i \leqq N_{1}-k$ where $0 \leqq k \leqq N_{1}-1$,

l) $C_{(0) r r, l l-s}^{w i-1}=C_{(0) r r-1, l l-s}^{w i-2}, C_{(0) r r, l l-s}^{w i-2}=0$

for $i \leqq N_{1}-s$ where $0 \leqq s \leqq N_{1}-1$,

m) $C_{(0) r r-i+1, l l-j+1}^{w N_{1}-k}=0$ for $k \geqq i+j$.

We give here only proofs of the more difficult points: a), d), f), g), and i):

We rewrite (19) in the following form:

$$
C_{(0) r s+1, l k+1}^{w i}-C_{(0) r s+1, l k}^{w i-1}=C_{(0) r s, l k+1}^{w i-1}-C_{(0) r s, l k}^{w i-2}
$$

treating this like a recurrence formula we obtain (i) for $i>s$, for $i \leqq s$ we obtain

$$
C_{(0) r s+1, l k+1}^{w i}-C_{(0) r s+1, l k}^{w i-1}=C_{(0) r s-i+2, l k+1}^{w 1} .
$$

${ }^{5}$ In the following $1 \leqq i \leqq N_{1}$ if not assumed other limitation. 
It vanishes from point (e) so we obtain new recurrence formula which gives us (f) for $i>k$ and (g) for $k \geqq i$.

$\mathbf{a d ~ h ) ~ a n d ~ j ) ~ P r o o f ~ i s ~ a n a l o g o u s ~ t o ~ t h e ~ a b o v e ~ g i v e n . ~}$

ad $\mathbf{k})$ : We prove this by induction over $k$ :

1. For $k=0, C_{(0) r r, l l}^{w i-1}=C_{(0) r r, l l-1}^{w i-2}, C_{(0) r r, l l}^{w i-2}=0$ we obtain this directly from (19) by putting

$$
s=r, k=l-1 \text { and } s=r, k=l \text {. }
$$

2. Let us assume that for $k=m: C_{(0) r r-m, l l}^{w i-1}=C_{(0) r r-m, l l-1}^{w i-2}$ $C_{(0) r r-m, l l}^{w i-2}=0$ for $i \leqq N_{1}-m$, on rewriting (19) for $s=r-m-1, k=l-1$ we obtain

$$
C_{(0) r r-m l l}^{w i-1}-C_{(0) r r-m, l l-1}^{w i-2}=C_{(0) r r-m-1, l l}^{w i-2}-C_{(0) r r-m-1, l l-1}^{w i-3}
$$

now if $i \leqq N_{1}-m$ then from inductive assumption we obtain

$$
C_{(0) r r-1}^{w i^{\prime}-1}(m+1), l l-C_{(0) r r-2}^{w i^{\prime}-2}(m+1), l l-1=0
$$

for $i^{\prime} \leqq N_{1}-(m+1)$, on rewriting (19) for $s=r-m-1, k=l$ we obtain $C_{(0) r r-(m+1)}^{w i^{\prime}-2}=0$ for $i^{\prime} \leqq N_{1}-(m+1)$ what ends our proof.

ad l): The proof is analogous to the given one for the point $(\mathrm{k})$.

ad $\mathbf{m})$ : We can prove this starting from $(\mathbf{k})$ or $(\mathbf{l})$. We start from (k). We take $C_{(0) r r-i+1, l l}^{w i^{\prime}-2}=0$ for $i^{\prime} \leqq N_{1}-(i-1)$. Now using first part of (k) as a recurrence formula we have $C_{(0) r r-i+1, l l-j+1}^{w i^{\prime}-2-j+1}=0$ for $i^{\prime} \leqq N-i+1$. Now we give the proofs of the corollaries:

ad 1.: We can prove this in one particular basis because these features of Lie algebra are basis independent. We see that in our basis the proof follows from the Eq. (18) and from the points (b) and (g).

ad 2.: In our basis it is equivalent to the point (m).

ad 3.: Follows immediately from (20) which implies that only $C_{(0) \alpha, \beta \neq 0}^{w w}$.

ad 4.: From (20) it follows that the $C_{(1) \alpha, \beta}^{\gamma}$ being a linear combination of $C_{(0) \alpha, \beta}^{\nu}$ satisfy the equations $(17-19)$, then also from (20) one can read that for $p>1 C_{(2) \alpha, \beta}^{\gamma}=0$, for $p=0$ one finds $C_{(1) \alpha, \beta}^{\gamma}$, the connection between $C_{(1) \alpha, \beta}^{\gamma}$ and $C_{(0) \alpha, \beta}^{\gamma}$ gives an inductive relation between $C_{(i) \alpha, \beta}^{\gamma}$ and $C_{(i-1) \alpha, \beta}^{\gamma}$ what allows to prove easily by induction relation (21). In the same way one proves $\left(21^{\prime}\right)$.

ad 5.: All $R^{(m)}$ can be contracted by $A(\varepsilon)$ so for each of them one can prove Corollary 1.

ad 6.: We have to prove the following equality $C_{(m) r r-i+1, l l-j+1}^{w s}=0$, for $m \geqq i, j$ and $s \leqq w-(i+j-m)$. To prove this we use $(21)$ and the point $(\boldsymbol{m})$ for $p=0$. From $(21)$ we obtain $C_{(m) r-i+1, l l-j+1}^{w i}=0$ for $i \leqq w-m, C_{(m) r r-i+1, l l-j+1}^{w w-c}=-C_{(0) r r-i+1+c, l l-j+1+c}^{w v-m+c}$ for $m \geqq i$ and $m \geqq j$, now from point $(\boldsymbol{m})$ for $p=0 \quad C_{(0) r r}^{w w-i+1+c, l l-j+1+c}=0$ for 
$(m-c) \geqq i+j-2 c$ it means for $c \geqq i+j-m$ which ends our proof, because from (21) easily follows that $C_{(m) r}^{n} \underset{k}{r-i+1, l l-j+1}=0$.

ad 7. : Follows from $(6)$ because $S_{k}=\bigoplus_{i=1}^{k} A_{i}$.

ad 8.: Follows from (7) and from (21) (where from using (b) one obtains $\left[S_{R}, S_{k}\right]^{(m)} \subset S_{k}$ for $\left.m \geqq k\right)$.

\section{The $(\boldsymbol{S}, \boldsymbol{p})$ Contractions for $\varepsilon_{0}=\infty$}

Now one can try to investigate a contraction by the same $A(\varepsilon)(15)$ but assuming $\varepsilon_{0}=\infty$, we will signify this contraction $(S, p)_{\infty}$. To find the necessary and sufficient conditions for $(S, p)_{\infty}$ one has to investigate convergence of the equalities (16) as $\varepsilon \rightarrow \infty$. We see that limit of (2), as $\varepsilon \rightarrow \infty$, exists if and only if:

$$
\begin{aligned}
C_{(0) s, t}^{n} & =\delta_{p 0} C_{(0) s, t}^{n}, \quad C_{(0) s, l k}^{w i}=\delta_{p 0} C_{(0) s, l k}^{w i} \\
C_{(0) s, t}^{w i} & =\left(\delta_{p 0}+\delta_{p 1}\right) C_{(0) s, t}^{w i}, \\
C_{(0) r s, l k}^{n} & =C_{(0) r s, l k}^{w i}=C_{(0) s, l k}^{n}=0 .
\end{aligned}
$$

New structure constants of $R^{(1)}$ can be found from (16) by the use of $(22)$ in the form:

$$
\begin{aligned}
C_{(1) s, t}^{n} & =C_{(0) s, t}^{n} \delta_{p 0}, \quad C_{(1) s, t}^{w i}=\delta_{p 1} C_{(0) s, t}^{n i}, \\
C_{(1) s, l k}^{w i} & =\delta_{p 0} C_{(0) s, l k}^{w i}, \\
C_{(1) r s, l k}^{n} & =C_{(1) r s, l k}^{w i}=C_{(1) s, l k}^{n}=0 .
\end{aligned}
$$

All these conditions can be expressed in a basis independent way:

Theorem 3. The algebra $R^{(0)}$ can be contracted by $(S, p)_{\infty}$ if and only if:

a) for $p>1-R^{(0)}$ is abelian,

b) for $p=1-\left[S_{R}, S_{R}\right]^{(0)} \subset S_{N}$ and $\left[S_{N}, S\right]^{(0)}=0$,

c) for $p=0-\left[S_{N}, S_{N}\right]^{(0)}=0$ and $\left[S_{N}, S_{R}\right]^{(0)} \subset S_{N}$.

Theorem 4. If we contract the algebra $R^{(0)}$ by $(S, p)_{\infty}$ we obtain a nonisomorphic algebra $R^{(1)}$ only for $p=0$. For $p=0, R^{(1)}$ satisfies the following commutation relations

$$
\left[S_{R}, S_{l l}\right]^{(1)}<S_{R},\left[S_{N}, S_{N}\right]^{(1)}=0,\left[S_{N}, S_{R}\right]^{(1)} \subset S_{N} .
$$

So $R^{(1)}$ is a semidirect product of subalgebra $G_{R}$ (generated by $S_{R}$ ) by an abelian ideal $A$ (generated by $S_{N}$ ) $R^{(1)}=A \in G_{R}$.

The proofs of the preceding theorems are trivial.

Corollary 9. To obtain $R^{(1)}=A \oplus G_{R}{ }^{*}$ by $(S, 0)_{\infty}$ we have to demand $R^{(0)}$ to satisfy the following condition $\left[S_{N}, S\right]^{(0)}=0$.

As shown by Doebner and Mersheimer [1] this special case is interesting from the physical point of view.

* $\oplus$ means here a direct product of algebras.

11 Commun. math. Phys., Vol. 13 


\title{
6. Discussion
}

So we obtained the necessary and sufficient conditions for $(S, p)$ and the brackets of $R^{(1)}$ expressed only by the structure constants of $R^{(0)}$. We hope that our equations $(17-20)$ are more handy than the equations $(9-12)$ and they do not involve the unknown matrix " $u$ ". We obtained 9 basis independent corollaries and 13 small corollaries expressed by the structure constants of $R^{(0)}$. Some of our corollaries were obtained for $p=0$ by Saletan. Saletan didn't obtain the corollaries 2, 3, $4,6,9$.

We hope that using our basis (13) one can try to investigate more general contraction for example by $A(\varepsilon)=\left(I \oplus u_{N}\right)+B(\varepsilon)$ where $B(\varepsilon)$ is a p-contraction.

We also investigated $(S, p)_{\infty}$ contractions but they turned out to be very restrictive and one obtains nontrivial results only for $p=0$.

Acknowledgement. The author is very indebted to Professor J. WerLe and to Dr. R. RACzKa for their valuable discussions.

\section{References}

1. Doebner, H. D., and O. Melshermer: Nuov. Cimento 49 A, 306 (1967).

2. Segal, S. I.: Duke Math. J. 18, 256 (1951).

3. Inonü, E., and E. P. Wigner: Proc. Natl. Acad. Sci. U.S. 39, 510 (1953).

4. Saletan, E. J.: J. Math. Phys. 2, 1 (1961).

5. Herman, R.: Commun. Math. Phys. 3, 53 (1966).

6. Levy-Nahas, M.: J. Math. Phys. 8, 1211 (1967).

7. -, and R. Seneor: Commun. Math. Phys. 9, 242 (1968).

8. Hegerfeld , G. C.: Nuovo Cimento, 51 A, 439 (1967).

9. Halmos, P. R.: Finite-dimensional vector space Princeton 1958.

\author{
Marian Kupczyński \\ Institute of Theoretical Physics \\ Warsaw University, \\ ul. Hoza 69 \\ Warsaw, Poland
}

